\section{Severity of TMD Related to Age, Sex and Electromyographic Analysis}

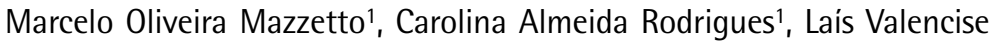
Magri', Melissa Oliveira Melchior ${ }^{1}$, Guiovaldo Paiva ${ }^{2}$

Temporomandibular disorders (TMD) are manifested as a group of signs and symptoms that affect a particular population profile. Some variables such as sex and age influence the clinical expression of this condition. This observational descriptive cross-sectional study aimed to correlate the severity of TMD established by the craniomandibular index (CMI) with the variables: age, sex and electromyographic activity of the masseter and anterior temporal muscles. Fifty-four subjects ( 15 males/39 females) aged between 16 to 65 years (mean age $=41$ years) and diagnosed with TMD were evaluated. Severity was determined by the CMI. These subjects also underwent examination by surface electromyography of the masseter and anterior temporal muscles. No correlation was found between age and severity of TMD $(p=0.19 / r=0.16)$, however there was a trend of greater severity in young adults (25-50 years). The sex variable in the correlation was positive with the CMI $(p=0.03 / r=-0.96)$ and superior to women. A greater EMG activity of the anterior temporal in relation to masseter $(p=0.01)$ was found and the left temporal activity had the highest average ( $161.5 \pm 44.6 \mathrm{~Hz})$. The electromyographic activity of the anterior temporal and right masseter muscles was positively correlated with the Dysfunction Index CMI ( $p=0.01)$. The use of CMI to quantify the severity of TMD and of EMG to assess the functionality of the masticatory muscles can be important allies to direct the treatment.

\author{
'Department of Restorative \\ Dentistry, Ribeirão Preto School of \\ Dentistry, USP - University of São \\ Paulo, Ribeirão Preto, SP, Brazil \\ ${ }^{2}$ Center for Diagnosis and \\ Treatment of Temporomandibular \\ Joint, São Paulo, SP, Brazil
}

Correspondence: Prof. Dr. Marcelo Oliveira Mazzetto, Avenida do Café S/N, 14040-904 Ribeirão Preto, SP, Brasil. Tel.:+55-16-3602-4087. e-mail: mazzetto@forp.usp.br

\section{Introduction}

Temporomandibular disorder (TMD) is characterized by a set of signs and symptoms, among them are pain in the temporomandibular joints, the preauricular and/or masticatory muscles, and limitation of mandibular mobility and tenderness (1).

Young adults aged between $20-40$ years are the most affected by this condition. The quality of life, stress factors and low adaptive capacity may explain why this age group is most affected. The literature has reported an increase of adolescents with TMD due to the same above described factors (2-4).

There is a documented epidemiological difference in the frequency and severity of TMD in females compared with males, as in females these indexes are higher probably due to the influence of behavioral, hormonal, anatomic and psychosocial causes. Women are three times more likely to develop myofascial pain compared with men $(2,5,6)$ and considering only the severe cases, may be about 9 times more affected than men (3).

Fricton and Schiffman (7) proposed a way to measure the severity of TMD by the Craniomandibular Index (CMI), which is composed by the dysfunction Index, related with mandibular mobility and joint sounds, and the palpation index which assesses pain sensitivity of the stomatognathic system structures (7).

Surface electromyography (EMG) has been established as an important method of analyzing muscle during orofacial behavior (8). The EMG measures the electric potential produced by individual masticatory muscles to verify and quantify the muscle balance between the muscles of both sides and between pairs of muscles, and evaluates muscle coordination in dynamic activities $(9,10)$.

It is possible to study the activity of the masticatory muscles by EMG (11) and determine the effects of TMD on these muscles during their functional movements (12). In subjects with TMD there is a change in muscle recruitment, which may be a compensatory mechanisms related to pain relief. This asymmetrical muscle recruitment may precede the development of muscular pain symptoms.

The aim of this study was to correlate the severity of TMD established by using the CMI with the variables: age, sex and EMG activity of the masseter and anterior temporal muscles.

\section{Material and Methods}

This observational cross-sectional descriptive study was approved by the Ethics Committee of the Ribeirão Preto School of Dentistry, University of São Paulo, under the protocol \#08982312.6.0000.5419.

Fifty-four subjects with a diagnosis of TMD were evaluated, being 15 males and 39 females with ages between 16 and 65 years (mean age $=41$ years). This enrollment was made from the data sheet used in our Occlusion Clinical Service for clinical examination (history, clinical evaluation, muscle and joint palpation). The 
evaluations were performed by a previously trained and calibrated dentist, specialist in TMD and orofacial pain.

Inclusion criteria were subjects diagnosed with muscular TMD, with at least 28 natural teeth either sound or with small restorations. Subjects wearing complete dentures, with less than 28 teeth, tumors, or who had suffered trauma or major surgery (grafts, orthognatic surgery) in the head and neck, or undergoing other treatments related to the stomatognathic system (dental care, speech therapy) were not accepted in the study.

The TMD severity in each subject was classified according to the CMI (7), which consists of the dysfunction index (DI) and the palpation index (PI) and encompasses 62 items coded as positive or negative. The DI evaluates the extent of TMJ dysfunction and quantifies jaw movement and joint sounds, such as clicks and crepitation. The PI evaluates the integrity of TMJ structures upon muscle palpation and quantifies the sensitivity associated with TMJ and orofacial and neck muscles (13). The total CMI score is the sum of the $\mathrm{DI}$ and PI scores divided by 2 . The scores of all 3 indices range from 0 (no dysfunction) to 1 (the most severe dysfunction), and a high score indicates a greater dysfunction in the TMJ and the surrounding muscles.

The subjects underwent surface electromyography (EMG) of the right anterior temporal (RT), left anterior temporal (LT), right masseter (RM) and left masseter (LM) muscles, using a Myotrace 400 (Noraxon, Scottsdale, AZ, USA) EMG equipment. EMG examinations were performed by another dentist, specialist in TMD and orofacial pain.

EMG readings and analyses were performed according to the Shewman's protocol (14), using bipolar silver/silver chloride surface electrodes (Hal Indústria e Comércio Ltda, São Paulo, SP, Brazil) with an available diameter of $10 \mathrm{~mm}$ and inter-electrode distance $21 \mathrm{~mm}$ (14). Thee electrodes were positioned on parallel fiber bundles of the examined muscles (RT, LT, RM and LM) and one reference electrode

Table 1. Pearson's correlation between the EMG activity of the masseter and anterior temporal left and right muscles with the palpation and dysfunction indexes

\begin{tabular}{lcc}
\hline Index x Muscle & p value & $\begin{array}{c}\text { Pearson's } \\
\text { coefficient (r) }\end{array}$ \\
\hline Palpation index x Right masseter & 0.70 & 0.06 \\
Palpation index x Left masseter & 0.78 & 0.04 \\
Palpation index x Right temporal & 0.30 & 0.15 \\
Palpation index x Left temporal & 0.85 & 0.03 \\
Dysfunction index x Right masseter & $0.01^{*}$ & 0.36 \\
Dysfunction index x Left masseter & 0.14 & 0.22 \\
Dysfunction index x Right temporal & $0.01^{*}$ & 0.38 \\
Dysfunction index x Left temporal & 0.08 & 0.26 \\
\hline
\end{tabular}

*Statistical significance by adopting $\alpha=0.05$. available to approximately $10 \mathrm{~mm}$ above the glabella, according to SENIAM (Surface EMG for Non-Invasive Assessment of Muscles) (15). The electromyographic examination was performed in 6 steps (13): Baseline/ Postural Evaluation - Seated; Baseline/Postural Evaluation - Standing; Functional Clench, Functional Control, Swallow and Long Clench. In all steps, the initial rest position was important to avoid the effect of any fatigue, and a rest period of at least 3 min was allowed between each test (16).

The variables (age, sex and electromyographic activity of temporal and masseter muscles) were correlated with the severity of TMD determined by the CMI and its components (DI and PI) using the D'Agostino-Pearson test of normality and Pearson's correlation test. The remaining analyses were performed using the Student's t-test, adopting a significance level of 5\%. The statistical software used for the analyses was the Bioestat 5.0.

\section{Results}

The mean $\mathrm{CMI}$ value was 0.48 , with values ranging from 0.13 to 0.77 . Figure 1 shows the CMI values according to age. No correlation was found between age and TMD severity, considering both the CMI ( $p=0.19$ ) (Table 1) and its components dysfunction and palpation indices $(p=0.84$, $p=0.08$, respectively).

With regard to males, $20 \%$ of subjects had mild TMD (CMI from 0 to 0.3 ), 60\% moderate TMD (CMI 0.3 to 0.6 ) and 20\% severe TMD (CMI 0.6 to 1). CMI distribution among females was $18 \%$ with mild TMD, 56\% moderate TMD and 26\% with severe TMD (Fig. 2). Analyzing the CMI no correlation was found between TMD severity and sex $(p=0.03 / r=-0.96)$, although there was a higher trend for the females. The analysis of the dysfunction and palpation indices showed no correlation either ( $p=0.37, p=0.30$, respectively).

The EMG examination of the anterior temporal and masseter muscles showed more activity of LT (161.5 \pm 44.6 Hz, mean and standard deviation) compared with RT

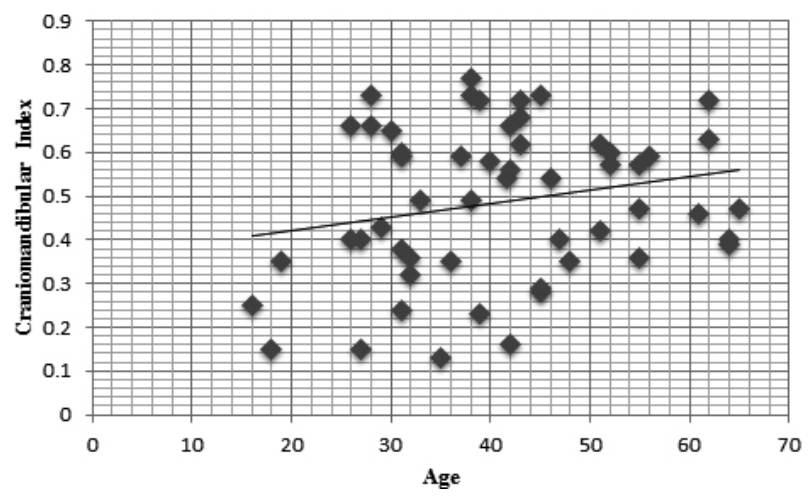

Figure 1. Severity of TMD established by craniomandibular index as a function of age. 
$(153.8 \pm 41.3 \mathrm{~Hz}), \mathrm{RM}(137.5 \pm 32.3 \mathrm{~Hz})$ and LM $(140 \pm$ $35.2 \mathrm{~Hz}$ ) (Fig. 3). In the same way, more EMG activity was found in the anterior temporal in relation to the masseter muscle $(p=0.01)$.

No correlation was found between the $\mathrm{CMI}$ and the mean of EMG activity of the evaluated muscles, considering the whole sample $(p=0.21 / r=0.17)$ or only the females $(n=39, p=0.29 / r=12.17)$. The correlations between the EMG activity of the analyzed muscles using $\mathrm{DI}$ and $\mathrm{PI}$ are presented in Table 1.

Positive statistical correlation was found between DI and EMG activity of the right anterior temporal and right masseter muscles ( $p=0.01$ ) (Figs. 4 and 5 ). No significant correlation was found for the other analyses ( $p>0.05$ ).

\section{Discussion}

Quality of life and stress have a direct influence on the prevalence of TMD according to age because young adults

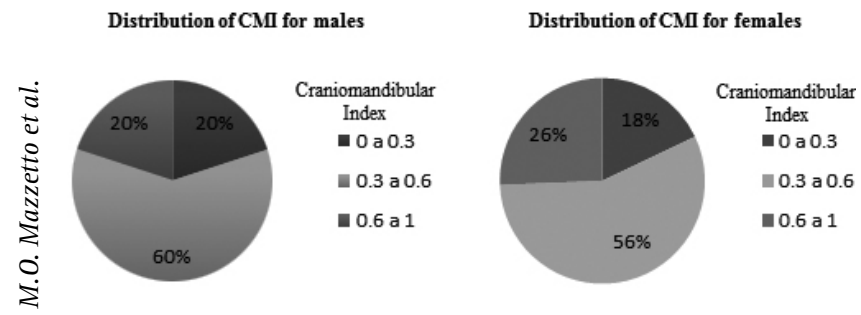

Figure 2. Severity of TMD established by craniomandibular index by sex.

Right Anterior Temporal

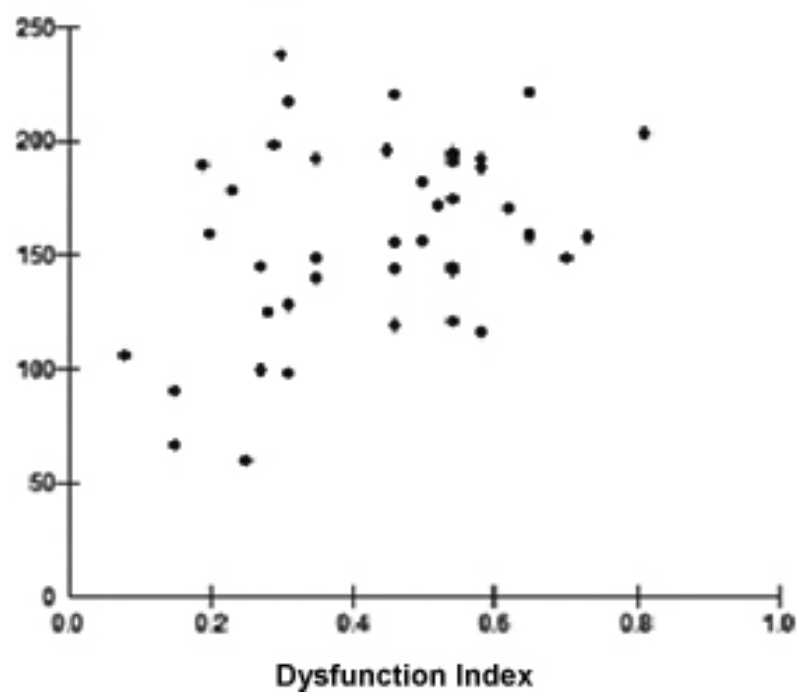

Figure 4. Correlation between the positive statistical significance with dysfunction index and electromyographic activity of the right anterior temporal muscle ( $\mathrm{p}=0.01$, Pearson's correlation test). are at the stage of life of greater production and distress (20). Advancing age produces changes in the standard of living and adaptive capacity of subjects with TMD, causing the signs and symptoms to become subclinical and perceived with less intensity or undetected, resulting in less pronounced severity.

The onset of TMD has become increasingly earlier. The literature shows that children and adolescents have been much more affected by this condition. Parafunctional

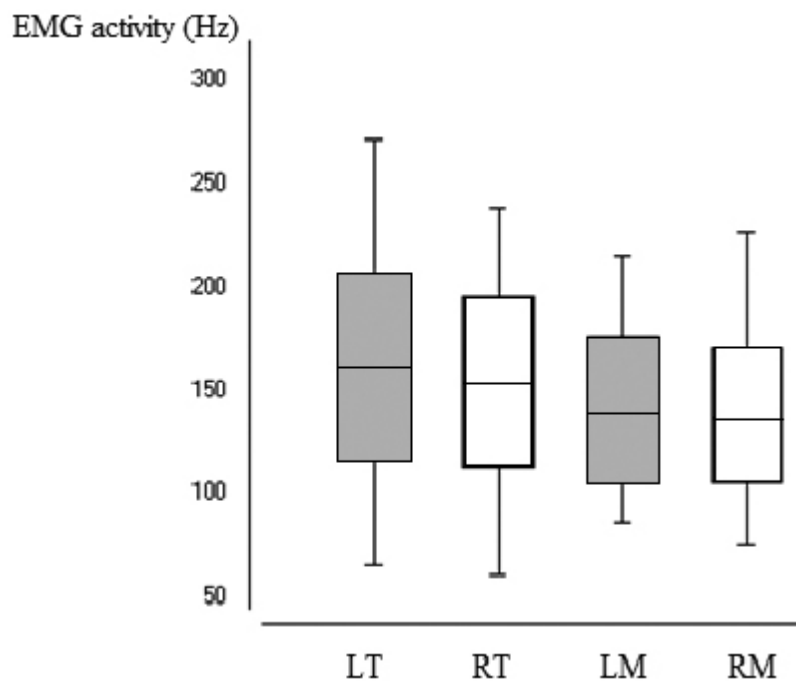

Figure 3. Mean and standard deviation of the electromyographic activity of the masseter and anterior temporal muscles. 
habits are seen as the main risk factors of TMD in children and adolescents (21).

Regarding the higher values for females when analyzing TMD severity, several studies have shown the existence of an epidemiological difference between males and females. Among women these rates are higher probably due to the influence of behavioral, hormonal, anatomic and psychosocial factors $(4,17,18)$. Women have a 3 -fold higher risk to develop myofascial pain than men, according to a prevalence study conducted with 83 patients (19). Thus, the results found in this study agree with other studies, which show that in women there is a greater involvement in relation to TMD, as well as increased expression of the signs and symptoms $(2,3,6)$.

Although the $\mathrm{CMI}$ allows for a quantitative assessment of the changes involving the TMD, it is not possible to establish a diagnosis based only in this index. It is necessary to include other assessment instruments with more established diagnostic criteria. Another limitation that may be attributed to the CMI is that the authors do not define cutoff values for categorizing the presence or absence of TMD. On the other hand, in a single clinical evaluation it is possible to establish the degree of involvement of structures affected by TMD (13), suggesting or not the need for application of other instruments designed for the diagnosis and classification of this condition. The use of $\mathrm{CMI}$ in conjunction with specific methods of diagnosis for determining the severity of the dysfunction has epidemiological importance in directing the therapeutic protocol.

The observed relationship of greater activity of the temporal muscles compared with masseter was also observed in other studies that used EMG as an evaluation method in subjects with TMD $(8,16)$. In previous studies, it was discussed that the muscle recruitment may be altered in subjects with TMD due to a compensatory mechanism for the relief of pain, or even precede the painful symptoms of masticatory muscles $(8,22)$. In this sense, Castroflorio et al. (23) stated that despite the EMG not being directly related to the diagnosis of orofacial pain, the technological advances in the detection and signal processing have enabled collecting information with quality, providing a better understanding of the anatomy and physiology of the stomatognathic system (23).

During research and clinical practice it is important to assess the severity of symptoms and signs, so the CMI was developed to provide a standardized measure of the severity of the changes related to ATM, including problems of mandibular movement, joint sounds, and joint and muscle sensitivity (7). There was no correlation between EMG and TMD severity measured by the CMI. However, the positive correlation found between this index and EMG dysfunction of the anterior temporal and right masseter, suggests that the functional consequences of the action of the muscles can be demonstrated by surface EMG according to the electrical activity of muscles. PI showed no correlation with the surface EMG of the examined muscles. The current understanding of orofacial pain becomes evident when it elucidates the idea that orofacial pain is related to a diagnosis on the sensitivity, which cannot be measured by electromyography $(1,6)$.

The pain and changes in jaw movements do not always present clinical relations, it is possible that the presence of pain without the mandibular function has changed, or there is a change in mandibular function, but no pain (8). Thus, as the EMG evaluates aspects related to the functional activity of the muscles it should never be used as the only means of diagnosis and should always be associated with other methods of evaluation, especially the history and clinical examination, which is mandatory in establishing the diagnosis of TMD (1).

Several studies highlighted the importance of understanding the relationship between pain and neuromuscular status, based on EMG analysis. It provides relevant functional information $(9,10,14,22)$, like the masticatory side preference (24), symmetry of activity before and after interventions (25), the functional impact of morphological discrepancies, among others (10).

In contrast with this study, De Felicio et al. (8) found a significant inverse correlation between EMG activity, considering the total average of the analyzed muscles (anterior temporal and masseter) and TMD severity perceived by the subjects (8). The composition of the sample, the research methodology, and especially the protocols that evaluated the severity of TMD, may explain the discordant results because De Felicio et al. (8) used the protocol ProDTMMulti with a numerical scale and symptomatic description broader than the CMI. Therefore, EMG has been valued as an additional method of diagnosis and its use and interpretation should be judicious, always allied to the clinical findings (8).

Within the methodological limitations of this study, full CMI correlated only with sex. Furthermore, the EMG activity was correlated to the functionality of the masticatory muscles, measured by the DI. This allowed for reasoning treatment needs to focus on strategies aimed at myofunctional recovery, given the diagnostic value of surface EMG. Using the CMI to quantify the severity of TMD and EMG to assess the functionality of the masticatory muscles can be an important approach in directing therapeutic management.

\section{Resumo}

As disfunções temporomandibulares (DTM) se manifestam como um 
conjunto de sinais e sintomas que acometem um determinado perfil da população. Algumas variáveis como gênero e idade interferem na expressão clínica desta condição. Este estudo observacional descritivo de corte transversal teve como objetivo correlacionar a severidade da DTM estabelecida pelo Índice Craniomandibular (ICM) com as variáveis: idade, gênero e atividade eletromiográfica dos músculos masseter e temporal anterior. Foram avaliados 54 (n) sujeitos ( 15 homens/39 mulheres), com idade entre 16 a 65 (idade média $=41$ anos), diagnosticados com DTM. A severidade foi determinada através do ICM. Estes sujeitos também foram submetidos ao exame de eletromiografia de superfície dos músculos masseter e temporal anterior. Não foi encontrada correlação entre a idade e a severidade da DTM ( $p=0.19 / r=0.16)$, todavia foi observada tendência de maior severidade em adultos jovens (25-50 anos). Na variável gênero a correlação foi positiva com o ICM ( $p=0.03 / r=-0.96)$, havendo superioridade para as mulheres. Foi encontrada maior atividade eletromiográfica dos temporais anteriores em relação aos masseteres $(p=0.01)$, sendo que o temporal esquerdo apresentou a maior atividade média $(161.5 \pm 44.6 \mathrm{~Hz})$. A atividade eletromiográfica dos músculos temporal anterior e masseter à direita foram correlacionados positivamente com o Índice de Disfunção $(p=0.01)$. A utilização do ICM para quantificar a severidade da DTM e a EMG para avaliar a funcionalidade dos músculos mastigatórios podem ser aliados importantes no direcionamento da conduta terapêutica.

\section{References}

1. Scrivani SJ, Keith DA, Kaban LB. Temporomandibular disorders. N Engl J Med 2008;359:2693-2705.

2. Manfredini D, Chiappe G, Bosco M. Research diagnostic criteria for Temporomandibular Disorders (RDC/TMD) axis I diagnoses in an Italian patient population. J Oral Rehab 2006;33:551-558.

3. Nomura K, Vitti M, Oliveira AMS, Chaves TC, Semprini M, Siéssere S, et al.. Use of the Fonseca's questionnaire to assess the prevalence and severity of temporomandibular disorders in Brazilian dental undergraduates. Braz Dent J 2007;18:163-167.

4. Nilsson IM, Drangsholt M, List T. Impact of temporomandibular disorder pain in adolescents: differences by age and gender. J Orofac Pain 2009;23:115-122.

5. Velly AM, Gornitsky M, Philippe P. Contributing factors to chronic myofascial pain: a case-control study. Pain 2003;104:491-499.

6. Poveda RR, Bagan JV, Díaz Fernandes JM, Hernández Bázan S, Jiménez Soriano Y. Review of temporomandibular joint pathology. Part I: classification, epidemiology and risk factors: review. Med Oral Patol Oral Cir Bucal 2007;12:292-298.

7. Fricton JR, Schiffman EL. Reliability of a Craniomandibular. J Dent Res 1986;65:1359-1364.

8. De Felício CM, Ferreira CLP, Medeiros APM, Da Silva MAMR, Tartaglia $\mathrm{GM}$, Sforza C. Electromyographic indices, orofacial myofunctional status and temporomandibular disorders severity: a correlation study. J Electromyogr Kinesiol 2012;22:266-272.

9. Ferrario VF, Sforza C. Coordinated electromyographic activity of the human masseter and temporalis anterior muscles during mastication. Eur J Oral Sci 1996;104:511-517.

10. Ferrario VF, Tartaglia GM, Maglione $M$, Simion M, Sforza C. Neuromuscular coordination of masticatory muscles in subjects with two types of implant-supported prostheses. Clin Oral Implants Res 2004;15:219-225.

11. Takada K, Miyawaki S, Tatsuda M. The effects of food consistency on jaw movement and posterior temporalis and inferior orbicularis oris muscle activities during chewing in children. Arch Oral Biol 1994:39:793-805.

12. Nishigawa $K$, Nakano $M$, Bando $E$. Study of jaw movement and masticatory muscle activity during unilateral chewing with and without balancing side molar contacts. J Oral Rehab 1997;24:691-696.

13. Hatch JP, Rugh JD, Sakai S, Prihoda TJ. Reliability of the Craniomandibular Index. J Orofac Pain 2002;16:284-295.

14. Shewman T. Surface electromyography in Temporomandibular Dysfunction: a beginner's guide to clinical applications. Advanced Aesthetic and Neuromuscular Solutions (AANMS). Las Vegas, NV 2006.

15. Hermens HJ, Frerics B, Disslhorst-Klug C, Rau G. Development of recommendations for SEMG sensors and sensor placement procedures. J Electromyogr Kinesiol 2000;10:361-374.

16. Tartaglia GM, Lodetti G, Paiva G, De Felício CM, Sforza C. Surface electromyographic assessment of patients with long lasting temporomandibular joint disorder pain. J Electromyogr Kinesiol 2011;21:659-664.

17. Manfredini D, Piccotti F, Ferronato G, Guarda-Nardini L. Age peaks of different RDC/TMD diagnoses in a patient population. J Dent 2010;38:392-399.

18. Slade GD, Bair E, Greenspan JD, Dubner R, Fillingim RB, Diatchenko L, Maixner W, Knott C, Ohrbach R. Sings and symptoms of first-onset TMD and sociodemographic predictors of its development: the OPPERA prospective cohort study. J Pain 2013;14:T20-T32.

19. Velly AM, Gornitsky M, Philippe P. Contributing factors to chronic myofascial pain: a case-control study. Pain 2003;104:491-499.

20. Barros V de M, Seraidarian PI, Côrtes MI, de Paula LV. The impact of orofacial pain on the quality of life of patients with temporomandibular disorder. J Orofac Pain 2009;23:28-37.

21. Cortese SG, Fridman DE, Farah CL, Bielsa F, Grinberg J, Biondi AM. Frequency of oral habits, dysfunctions, and personality traits in bruxing and nonbruxing children: a comparative study. J Craniomand Pract 2013;31:283-290.

22. De Felício CM, Mapello A, Sidequersky FV, Tartaglia GM, Sforza C. Mandibular kinematics and mastigatory muscles EMG in patients with short lasting TMD of mild-moderate severity. J Electromuogr Kinesiol 2013;23:627-633.

23. Castroflorio T, Bracco P, Farina D. Surface electromyography in the assessment of jaw elevator muscles. J Oral Rehab 2008;35:638-645.

24. Zamanlu M, Khamnei S, Salarilak S, Oskoee SS, Shakouri SK, Houshyar $Y$, et al.. Chewing side preference in first and all mastication cycles for hard and soft morsels. Int J Clin Exp Med 2012;5:326-331.

25. Ferrario VF, Sforza C, Tartaglia GM, Dellavia C. Immediate effect of a stabilization splint on masticatory muscle activity in temporomandibular disorder patients. J Oral Rehab 2002;29:810-815.

Received August 23, 2013

Accepted January 28, 2014 